\title{
Mathematical problem with exact lump solutions in SFT
}

\section{Loriano Bonora *广}

SISSA, Via Bonomea 265, 34136 Trieste, Italy

and INFN, Sezione di Trieste

E-mail: bonora@sissa.it

\section{Stefano Giaccari ${ }^{\ddagger}$}

SISSA, Via Bonomea 265, 34136 Trieste, Italy

and INFN, Sezione di Trieste

E-mail: giaccari@sissa.it

\section{Driba D. Tolla ${ }^{\S}$}

Department of Physics and University College,

Sungkyunkwan University, Suwon 440-746, South Korea

E-mail: dribatolla@gmail.com

In this paper we analyze some mathematical problems that arise in the search for lump solutions in bosonic string field theory. We closely analyse the would-be violation of the equation of motion for such solutions that has been pointed out in the literature. We argue that, when the issue is considered in the appropriate mathematical setting of distribution theory, no violations of the equation of motion occur.

7th Conference Mathematical Methods in Physics - Londrina 2012,

16 to 20 April 2012

Rio de Janeiro, Brazil

\footnotetext{
* Speaker.

${ }^{\dagger}$ L.B would like to thank Nobuyuki Ishibashi and Branko Dragovic for useful discussions. The work of L.B. was supported in part by the MIUR-PRIN contract 2009-KHZKRX.

$\ddagger$ The work of S.G. was supported in part by the MIUR-PRIN contract 2009-KHZKRX.

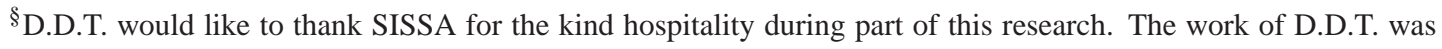
supported by the Korean Research Foundation Grant funded by the Korean Government with grant number KRF 20090077423.
} 


\section{Introduction}

Recently, following an earlier suggestion of [15], a general method has been proposed, [1], to obtain new exact analytic solutions in Witten's cubic open string field theory (OSFT) [5], and in particular solutions that describe inhomogeneous tachyon condensation. On general grounds it is expected that an OSFT defined on a particular boundary conformal field theory (BCFT) has classical solutions describing other boundary conformal field theories [6, 7]. Analytic solutions have actually been constructed describing the tachyon vacuum $[8,16,9]$ see also the reviews $[11$, 12]. In this panorama solutions describing inhomogeneous and relevant boundary deformations of the initial BCFT were not known until recently, though their existence was predicted $[6,7]$. This absence was filled up in [15, 1], and in [2, 3] the energy of a D24-brane solution was calculated for the first time. In [4] these results were extended to analytic SFT solutions corresponding to $\mathrm{D}(25-p)$-branes, for any $p$, and their energy was calculated.

Notwithstanding these successes, some formal problems have remained behind. Here we would like to discuss these problems. The first issue concerns the interpretation of the $\varepsilon$ parameter used in $[2,3,4]$. The latter was first introduced in the analysis as a regulator and subsequently (erroneously) interpreted as a gauge parameter, in the sense that physical quantities were supposed to be independent of it. Below we show that, both from a theoretical and a numerical point of view, $\varepsilon$ is a mere regulator (not a gauge parameter) and the only meaningful results are obtained in the $\varepsilon \rightarrow 0$ limit. This conclusion does not affect the results in $[2,4]$, because they were obtained precisely in that limit, but it clarifies a theoretical issue which is important in itself and also in relation to the subsequent point.

The next problem was raised in [3] and a solution to it was proposed in [2] in appendix D. In this note we would like to return to this issue and discuss it in full detail. It concerns a would-be violation of the SFT equation of motion for the string field candidates considered in $[1,2,4,3]$, which originates from the use of a Schwinger parametrization of inverse elements. Our discussion of the problem starts with pointing out that similar problems arise in the search for solutions in classical field theory. We show, for instance, that were we to take into account terms like the violating term of [3] (which we call spurious terms) in solving Einstein gravity in vacuum, we would come to the paradoxical conclusion that the Schwarzschild black hole is not a solution of Einstein gravity. We argue that, when the issue is considered in the proper setting, no violations to the equation of motion occur for the solutions considered in $[2,4]$. The spurious terms when inserted in convergent integrals give vanishing contributions and, on the other hand, can give nonvanishing (but ambiguous) contributions only if inserted in discontinuous integrals (see below for the precise meaning). This suggests that the appropriate mathematical setting to interpret them is the theory of distributions. We suggest that the lump solutions must be considered as distributions. Once this is done, any ambiguity linked to spurious terms in the equation of motion, disappears.

The paper is organized as follows. After a review of [1] and [2], section 2 is devoted to the clarification of the nature of the $\varepsilon$ parameter and relevant numerical calculations. In section 3 we outline the problem that arises when we represent $\frac{1}{K+\phi_{u}}$ by means of a Schwinger parametrization. In section 4 we discuss in detail the example of the Schwarzschild solution in Einstein gravity and show what would happen if we took into account spurious terms. In section 5 we argue on a general ground that the offending term of [3] does not have right of citizenship among well 
behaved mathematical objects. In section 6 we compute the offending (or spurious) term in the SFT equation of motion and show that it is in fact related to an ambiguity in the formalism, and should not be considered as a matter of principle, but, anyhow, even if taken into account, when inserted in a convergent expression for the energy, this term gives a vanishing contribution. In section 7 we introduces a numerous set of states that can play the role of test states in the distribution theory interpretation of the lump solution. Section 8 is devoted to a summary of the discussion and results. We also suggest that an appropriate mathematical framework for the problem discussed in this paper and for similar problems may be based on a re-elaboration of vector distribution theory.

\subsection{Review of the previous results}

In [1], to start with, the well-known $K, B, c$ algebra defined by

$$
K=\frac{\pi}{2} K_{1}^{L}|I\rangle, \quad B=\frac{\pi}{2} B_{1}^{L}|I\rangle, \quad c=c\left(\frac{1}{2}\right)|I\rangle,
$$

was enlarged as follows. In the sliver frame (obtained by mapping the UHP to an infinite cylinder $C_{2}$ of circumference 2 , by the sliver map $\tilde{z}=\frac{2}{\pi} \arctan z$ ), by adding a (relevant) matter operator

$$
\phi=\phi\left(\frac{1}{2}\right)|I\rangle
$$

with the properties

$$
[c, \phi]=0, \quad[B, \phi]=0, \quad[K, \phi]=\partial \phi,
$$

In this new algebra $Q$ has the following action:

$$
Q \phi=c \partial \phi+\partial c \delta \phi
$$

It can be easily proven that

$$
\psi_{\phi}=c \phi-\frac{1}{K+\phi}(\phi-\delta \phi) B c \partial c
$$

does indeed satisfy the OSFT equation of motion

$$
Q \psi_{\phi}+\psi_{\phi} \psi_{\phi}=0
$$

It is clear that (1.5) is a deformation of the Erler-Schnabl solution, see [10], which can be recovered for $\phi=1$.

In order to prove that (1.5) is a solution, one demands that $(c \phi)^{2}=0$, which requires the OPE of $\phi$ at nearby points to be not too singular.

Using the $K, B, c, \phi$ algebra one can show that

$$
\mathscr{Q}_{\psi_{\phi}} \frac{B}{K+\phi}=Q \frac{B}{K+\phi}+\left\{\psi_{\phi}, \frac{B}{K+\phi}\right\}=1 .
$$

So, unless the homotopy-field $\frac{B}{K+\phi}$ is singular, the solution has trivial cohomology, which is the defining property of the tachyon vacuum $[15,16]$. On the other hand, in order for the solution to be well defined, the quantity $\frac{1}{K+\phi}(\phi-\delta \phi)$ should be well defined too. Finally, in order to be able to show that (1.5) satisfies the equation of motion, one needs $K+\phi$ to be invertible.

In full generality we thus have a new nontrivial solution if 
1. $\frac{1}{K+\phi}$ is singular, but

2. $\frac{1}{K+\phi}(\phi-\delta \phi)$ is regular and

3. $\frac{1}{K+\phi}(K+\phi)=1$.

In [1] sufficient conditions for $\phi$ to comply with the first two requirements were determined. Let us parametrize the worldsheet RG flow, referred to above, by a parameter $u$, where $u=0$ represents the UV and $u=\infty$ the IR, and rewrite $\phi$ as $\phi_{u}$, with $\phi_{u=0}=0$. Then we require for $\phi_{u}$ the following properties under the coordinate rescaling $f_{t}(z)=\frac{z}{t}$

$$
f_{t} \circ \phi_{u}(z)=\frac{1}{t} \phi_{t u}\left(\frac{z}{t}\right)
$$

and, most important, that the partition function

$$
g(u) \equiv \operatorname{Tr}\left[e^{-\left(K+\phi_{u}\right)}\right]=\left\langle e^{-\int_{0}^{1} d s \phi_{u}(s)}\right\rangle_{C_{1}},
$$

satisfies the asymptotic finiteness condition

$$
\lim _{u \rightarrow \infty}\left\langle e^{-\int_{0}^{1} d s \phi_{u}(s)}\right\rangle_{C_{1}}=\text { finite. }
$$

It was pointed out in [1] that this satisfies the first two conditions above i.e. guarantees not only the regularity of the solution but also its 'non-triviality', in the sense that if this condition is satisfied, it cannot fall in the same class as the ES tachyon vacuum solution. It would seem that the last condition above cannot be satisfied in view of the first. But this is not the case. This is the main issue discussed in sec.3,5-8.

We will consider in the sequel a specific relevant operator $\phi_{u}$ and the corresponding SFT solution. This operator generates an exact RG flow studied by Witten in [13], see also [14], and is based on the operator (defined in the cylinder $C_{T}$ of width $T$ in the arctan frame)

$$
\phi_{u}(s)=u\left(X^{2}(s)+2 \ln u+2 A\right),
$$

where $A$ is a constant first introduced in [15]. In $C_{1}$ we have

$$
\phi_{u}(s)=u\left(X^{2}(s)+2 \ln T u+2 A\right)
$$

and on the unit disk $D$,

$$
\phi_{u}(\theta)=u\left(X^{2}(\theta)+2 \ln \frac{T u}{2 \pi}+2 A\right) .
$$

If we set

$$
g_{A}(u)=\left\langle e^{-\int_{0}^{1} d s \phi_{u}(s)}\right\rangle_{C_{1}}
$$

we have

$$
g_{A}(u)=\left\langle e^{-\frac{1}{2 \pi} \int_{0}^{2 \pi} d \theta u\left(X^{2}(\theta)+2 \ln \frac{u}{2 \pi}+2 A\right)}\right\rangle_{D}
$$


According to [13],

$$
g_{A}(u)=Z(2 u) e^{-2 u\left(\ln \frac{u}{2 \pi}+A\right)}
$$

where

$$
Z(u)=\frac{1}{\sqrt{2 \pi}} \sqrt{u} \Gamma(u) e^{\gamma u}
$$

Requiring finiteness for $u \rightarrow \infty$ we get $A=\gamma-1+\ln 4 \pi$, which implies

$$
g_{A}(u) \equiv g(u)=\frac{1}{\sqrt{2 \pi}} \sqrt{2 u} \Gamma(2 u) e^{2 u(1-\ln (2 u))}
$$

and

$$
\lim _{u \rightarrow \infty} g(u)=1 .
$$

Moreover, as it turns out, $\delta \phi_{u}=-2 u$, and so:

$$
\phi_{u}-\delta \phi_{u}=u \partial_{u} \phi_{u}(s)
$$

Therefore the $\phi_{u}$ just introduced satisfies all the required properties and consequently $\psi_{u} \equiv \psi_{\phi_{u}}$ must represent a D24 brane solution.

In I the expression for the energy of the lump solution was determined by evaluating a threepoint function on the cylinder $C_{T}$ of circumference $T$ in the $\arctan$ frame. It is given by

$$
\begin{aligned}
E\left[\psi_{u}\right]= & -\frac{1}{6}\left\langle\psi_{u} \psi_{u} \psi_{u}\right\rangle \\
= & \frac{1}{6} \int_{0}^{\infty} d(2 u T)(2 u T)^{2} \int_{0}^{1} d y \int_{0}^{y} d x \frac{4}{\pi} \sin \pi x \sin \pi y \sin \pi(x-y) \\
& \cdot g(u T)\left\{-\left(\frac{\partial_{2 u T} g(u T)}{g(u T)}\right)^{3}+G_{2 u T}(2 \pi x) G_{2 u T}(2 \pi(x-y)) G_{2 u T}(2 \pi y)\right. \\
& \left.-\frac{1}{2}\left(\frac{\partial_{2 u T} g(u T)}{g(u T)}\right)\left(G_{2 u T}^{2}(2 \pi x)+G_{2 u T}^{2}(2 \pi(x-y))+G_{2 u T}^{2}(2 \pi y)\right)\right\} .
\end{aligned}
$$

where $G_{u}(\theta)$ represents the correlator on the boundary, first determined by Witten, [13]:

$$
G_{u}(\theta)=\frac{1}{u}+2 \sum_{k=1}^{\infty} \frac{\cos (k \theta)}{k+u}
$$

Moreover $\mathscr{E}_{0}\left(t_{1}, t_{2}, t_{3}\right)$ represents the ghost three-point function in $C_{T}$.

$$
\mathscr{E}_{0}\left(t_{1}, t_{2}, t_{3}\right)=\left\langle B c \partial c\left(t_{1}+t_{2}\right) \partial c\left(t_{1}\right) \partial c(0)\right\rangle_{C_{T}}=-\frac{4}{\pi} \sin \frac{\pi t_{1}}{T} \sin \frac{\pi\left(t_{1}+t_{2}\right)}{T} \sin \frac{\pi t_{2}}{T} .
$$

Finally, to get (1.19) a change of variables $\left(t_{1}, t_{2}, t_{3}\right) \rightarrow(T, x, y)$, where

$$
x=\frac{t_{2}}{T}, \quad y=1-\frac{t_{1}}{T} .
$$


is needed.

The expression (1.19) has been evaluated in [2]. As it turns out, this expression has a UV ( $s \approx 0$, setting $s=2 u T$ ) singularity, which must be subtracted away. Therefore the result one obtains in general will depend on this subtraction*. In [2] it has been pointed out that a physical significance can be assigned only to a subtraction-independent quantity, and it has been shown how to define and evaluate such a quantity. First a new solution to the EOM, depending on a regulator $\varepsilon$, has been introduced ${ }^{\dagger}$

$$
\psi_{u}^{\varepsilon}=c\left(\phi_{u}+\varepsilon\right)-\frac{1}{K+\phi_{u}+\varepsilon}\left(\phi_{u}+\varepsilon-\delta \phi_{u}\right) B c \partial c .
$$

its energy being 0 (after the same UV subtraction as in the previous case) in the $\varepsilon \rightarrow 0$ limit. Then, using it, a solution to the EOM at the tachyon condensation vacuum has been obtained. The equation of motion at the tachyon vacuum is

$$
\mathscr{Q} \Phi+\Phi \Phi=0, \quad \text { where } \mathscr{Q} \Phi=Q \Phi+\psi_{u}^{\varepsilon} \Phi+\Phi \psi_{u}^{\varepsilon} \text {. }
$$

One can easily show that

$$
\Phi_{0}^{\varepsilon}=\psi_{u}-\psi_{u}^{\varepsilon}
$$

is a solution to (1.23). The action at the tachyon vacuum is $-\frac{1}{2}\langle\mathscr{Q} \Phi, \Phi\rangle-\frac{1}{3}\langle\Phi, \Phi \Phi\rangle$. Thus the energy of of the lump, $E\left[\Phi_{0}\right]$, is

$$
\begin{aligned}
E\left[\Phi_{0}\right] & =-\lim _{\varepsilon \rightarrow 0} \frac{1}{6}\left\langle\Phi_{0}^{\varepsilon}, \Phi_{0}^{\varepsilon} \Phi_{0}^{\varepsilon}\right\rangle \\
& =-\frac{1}{6} \lim _{\varepsilon \rightarrow 0}\left[\left\langle\psi_{u}, \psi_{u} \psi_{u}\right\rangle-\left\langle\psi_{u}^{\varepsilon}, \psi_{u}^{\varepsilon} \psi_{u}^{\varepsilon}\right\rangle-3\left\langle\psi_{u}^{\varepsilon}, \psi_{u} \psi_{u}\right\rangle+3\left\langle\psi_{u}, \psi_{u}^{\varepsilon} \psi_{u}^{\varepsilon}\right\rangle\right]
\end{aligned}
$$

The integrals in the four correlators at the RHS, are IR $(s \rightarrow \infty)$ convergent. The UV subtractions necessary for each correlator are always the same, therefore they cancel out. In [2], after UV subtraction, we obtained

$$
\begin{aligned}
& -\frac{1}{6}\left\langle\psi_{u}, \psi_{u} \psi_{u}\right\rangle=\alpha+\beta, \quad \lim _{\varepsilon \rightarrow 0}\left\langle\psi_{u}^{\varepsilon}, \psi_{u}^{\varepsilon} \psi_{u}^{\varepsilon}\right\rangle=0 \\
& \frac{1}{6} \lim _{\varepsilon \rightarrow 0}\left\langle\psi_{u}^{\varepsilon}, \psi_{u} \psi_{u}\right\rangle=\alpha-\frac{2}{3} \beta, \quad \frac{1}{6} \lim _{\varepsilon \rightarrow 0}\left\langle\psi_{u}, \psi_{u}^{\varepsilon} \psi_{u}^{\varepsilon}\right\rangle=\alpha-\frac{1}{3} \beta
\end{aligned}
$$

where $\alpha+\beta \approx 0.068925^{\ddagger}$ was evaluated numerically and $\alpha=\frac{1}{2 \pi^{2}}$ was calculated analytically. So $E\left[\Phi_{0}\right]=\alpha$ turns out to be precisely the D24-brane energy. In [4] the same result was extended to any Dp-brane lump.

${ }^{*}$ The subtraction does not fix by itself the zero-point energy. For instance, in the examples of [4], the expression corresponding to (1.19) is explicitly gauge dependent.

${ }^{\dagger}$ In [2] $\psi_{u}^{\varepsilon}$ was called $\psi_{\varepsilon}$.

${ }^{\ddagger}$ This number represents the result of an improved numerical evaluation and differs from the value given in [2] by 6 per mil. 


\section{Nature of the $\varepsilon$ parameter}

Eq.(1.25) and (1.26) is really what we proved in $[2,4]$. That is, the result we obtained is only valid in the limit $\varepsilon \rightarrow 0$, and this was the correct thing to do. However we were mislead by a wrong theoretical prejudice and by a too rough numerical result into believing that the expression in square brackets in the RHS of (1.25) is independent of $\varepsilon$, and therefore $\varepsilon$ can be interpreted as a gauge parameter. This is not the case, as we will show in this section: $\varepsilon$ is a simple regulator and physical quantities can be recovered only in the $\varepsilon \rightarrow 0$ limit.

In [2] we computed numerically $\left\langle\psi_{u}^{\varepsilon}, \psi_{u}^{\varepsilon} \psi_{u}^{\varepsilon}\right\rangle$, after making the necessary UV subtraction. The result was reported in Table 3 there, and led us to the idea that that is evidence of the analytic result being 0 for any $\varepsilon$. This convinced us that $\varepsilon$ is a gauge parameter and, as a consequence, also the full expression in square brackets in the RHS of (1.25) should not depend on $\varepsilon$. Although it did not have any practical consequence on the final result, it must be said that this is not true. The present section is devoted to clarifying this issue.

Let us deal first with $\left\langle\psi_{u}, \psi_{u} \psi_{u}\right\rangle-\left\langle\psi_{u}^{\varepsilon}, \psi_{u}^{\varepsilon} \psi_{u}^{\varepsilon}\right\rangle$. One of the limits of the numerical evaluation of $\left\langle\psi_{u}^{\varepsilon}, \psi_{u}^{\varepsilon} \psi_{u}^{\varepsilon}\right\rangle$ in [2] was that the numerics can start only after the UV subtraction is carried out. This limits considerably the accuracy of the numerical approximation. The expression

$$
\Delta_{\varepsilon}^{(1)}=\left\langle\psi_{u}, \psi_{u} \psi_{u}\right\rangle-\left\langle\psi_{u}^{\varepsilon}, \psi_{u}^{\varepsilon} \psi_{u}^{\varepsilon}\right\rangle
$$

instead, is UV finite and its numerical evaluation can be more accurate. Here we report the numerical results for a sample of values of the parameter $\eta=\frac{\varepsilon}{2 u}$.

$\begin{array}{rcccccc}\eta: & 2 & 1 & 0.7 & 0.5 & 0.1 & 0.08 \\ \Delta_{\varepsilon}^{(1)}: & -0.41968 & -0.41958 & -0.42028 & -0.41860 & -0.41868 & -0.41853 \\ & & & & & & \\ \eta: & 0.05 & 0.01 & 0.005 & 0.003 & 0.001 & 0.0005 \\ \Delta_{\varepsilon}^{(1)}: & -0.41831 & -0.41660 & -0.41625 & -0.41587 & -0.41483 & -0.414009\end{array}$

Table 1: Samples of $\Delta_{\varepsilon}^{(1)}$

The limit $\lim _{\mathcal{E} \rightarrow 0} \Delta_{\varepsilon}^{(1)}$ was calculated in [2] and is given by: $6(\alpha+\beta) \approx-0.41355$. Since the numbers in Table 1 are accurate up to the third digit (being very conservative the error can be estimated to be \pm 0.0005 ) the dependence on $\varepsilon$ is evident. It is also clearly visible that the sequence of numbers tends to the expected value (around $\eta=0.00001$ reliable numerical results becomes hard to retrieve). The smallness of the $\varepsilon$ dependence (a few percent only) was at the origin of the misunderstanding about the nature of $\varepsilon$.

The dependence on $\varepsilon$ of

$$
\Delta_{\varepsilon}^{(2)}=\left\langle\psi_{u}^{\varepsilon}, \psi_{u} \psi_{u}\right\rangle-\left\langle\psi_{u}, \psi_{u}^{\varepsilon} \psi_{u}^{\varepsilon}\right\rangle
$$

is not much easier to detect. In Table 2 we report the numerical results for a sample of the parameter $\eta$.

In [2] the numerical value of $\Delta_{\varepsilon}^{(2)}$ was determined in the $\varepsilon \rightarrow 0$ limit to be: $\lim _{\varepsilon \rightarrow 0} \Delta_{\varepsilon}^{(2)}=$ $-2 \beta \approx-0.03652$. The results in Table 1 are to be taken with a possible uncertainty of \pm 0.0005 . We see that they clearly depend on $\varepsilon$ and that the limit $\varepsilon \rightarrow 0$ tends to the expected value. 


$\begin{array}{cccccc}\eta: & 10 & 2 & 1 & 0.7 & 0.5 \\ \Delta_{\varepsilon}^{(2)}: & -0.01431 & -0.02704 & -0.0308524 & -0.0323693 & -0.03332 \\ & & & & & \\ \eta: & 0.4 & 0.2 & 0.1 & 0.08 & 0.05 \\ \Delta_{\varepsilon}^{(2)}: & -0.03398 & -0.03525 & -0.03567 & -0.03550 & -0.03613\end{array}$

Table 2: Samples of $\Delta_{\varepsilon}^{(2)}$

After these results the dependence on $\varepsilon$ of (1.25) needs not be stressed. $\varepsilon$ is no gauge parame$t e r$, it is a simple regulator, as it was originally conceived. This conclusion could have been reached from a theoretical point of view. We see in fact that, while our $\phi_{u}(s)$ satisfies condition (1.7), the combination $\phi_{u}(s)+\varepsilon$, which appears in $\psi_{u}^{\varepsilon}$, does not since:

$$
f_{t} \circ \varepsilon=\varepsilon \neq \frac{\varepsilon}{t}
$$

Forcing $\varepsilon$ to satisfy (1.7) would require $\varepsilon=\kappa u$ for some positive constant $\kappa$; but then, in $\phi_{u}+\varepsilon$, see (1.10), $\varepsilon$ could be absorbed into a redefinition of $A$ and would disappear from $\phi_{u}^{\varepsilon}$. As a consequence the latter would actually coincide with $\psi_{u}$ and $\Delta_{\varepsilon}^{(1)}$ would vanish, which is evidently not the case. The role of $\varepsilon$ is precisely to break the covariance under the semigroup of rescalings, eq.(1.7), in order to generate a different kind of solution with respect to $\psi_{u}$. The conclusion is that the parameter $\varepsilon$ does not run (in the RG parlance), therefore it is not a gauge parameter (in the SFT terminology). We remark that the value $\varepsilon=0$ is (together with $\varepsilon=\infty$ ) the only scale invariant one.

One may be surprised at first that $\psi_{u}^{\varepsilon}$ is a solution to the EOM of SFT, while the term $\left\langle\psi_{u}^{\varepsilon}, \psi_{u}^{\varepsilon} \psi_{u}^{\varepsilon}\right\rangle$ is $\varepsilon$-dependent. The point is that $\psi_{u}^{\varepsilon}$ formally solves the equation of motion but is not an extreme of the action for $\varepsilon \neq 0$. The puzzle is explained of course by the fact that the parameter $\varepsilon$ is not present in the original action. Therefore one has to prove a posteriori that the 'solution' actually corresponds to an extreme of the action ${ }^{\S}$. The variation of the action with $\varepsilon$ is given by (after replacing the eom) $\delta_{\varepsilon} S \sim\left\langle\frac{\partial \psi_{u}^{\varepsilon}}{\partial \varepsilon}, Q \psi_{u}^{\varepsilon}\right\rangle-\left\langle Q \frac{\partial \psi_{u}^{\varepsilon}}{\partial \varepsilon}, \psi_{u}^{\varepsilon}\right\rangle$. For this to vanish one should be able to 'integrate by parts', which is not possible due to the UV subtractions implicit in the calculation of the correlators, see [2] (and also [18] where similar arguments are developed although not in the same context) ${ }^{\pi}$. Now $\delta_{\varepsilon} S$ does not vanish and in order to find an extreme of the action we have to extremize it. This is in keeping with the monotonic dependence on (small) $\eta$ one can see in Table 1 , which tells us that the extreme is met in the limit $\varepsilon \rightarrow 0$.

We have verified that also other quantities considered in section 6 of this paper, which contain $\varepsilon$, are effectively $\varepsilon$-dependent. In the light of the above theoretical argument, this and the previous numerical proof that $\Delta_{\varepsilon}^{(1)}$ and $\Delta_{\varepsilon}^{(2)}$ are $\varepsilon$-dependent would be pointless, if a misunderstanding about the role of $\varepsilon$ had not arisen. In any case, having at hand Table 1 and 2 , we have the opportunity to

\footnotetext{
${ }^{\S}$ The same consideration applies also to the parameter $u$, but it was shown in [1] that $u$ actually disappears from the action when we replace $\psi_{u}$ in it: $u$ is a true gauge parameter.

I Since the UV singularity is linked to the $X$ zero mode, one might expect that with a compactified $X$ this problem should disappear and the integration by parts become possible. However, as long as we consider solution of the type $\psi_{u}, \psi_{u}^{\varepsilon}$ with a linearly scaling $u$ parameter, this seems to be impossible: the singularity removed from the UV will pop up in the IR, creating analogous problems. The nontrivial boundary contribution in the SFT action, see also section 2, is a new interesting feature which deserves a closer investigation.
} 
make the following observation: the limit $\varepsilon \rightarrow 0$ is smooth and it tends to the expected theoretical value (just replace the numerical values of $\Delta_{\varepsilon}^{(1)}$ and $\Delta_{\varepsilon}^{(2)}$ inside (1.25)). Nothing anomalous happens in the limit. We have proven the existence of the limit also analytically (we will spare the reader the lengthy details), but the numerical results are more pictorial. Eq.(1.25) was obtained by plugging in $\mathscr{Q} \Phi_{0}=-\Phi_{0} \Phi_{0}$ into the SFT action. Should the EOM be violated, the worst that can happen is that the violating term, if any, contributes 0 to the energy. This is exactly what we will show in section 6 .

\section{The problem with the Schwinger representation}

We now come to the criticism raised by [3] about our solution. In order to obtain (1.19) one has to use the following Schwinger representation

$$
\frac{1}{K+\phi_{u}}=\int_{0}^{\infty} d t e^{-t\left(K+\phi_{u}\right)}
$$

of the inverse of $K+\phi_{u}$. When using such a Schwinger representation, however, the identity

$$
\frac{1}{K+\phi_{u}}\left(K+\phi_{u}\right)=I
$$

would seem not to be satisfied. To illustrate the problem, let us calculate the overlap of both the left and the right hand sides of (3.2) with $Y=\frac{1}{2} \partial^{2} c \partial c c$. The right hand side is trivial and, in our normalization, it is

$$
\operatorname{Tr}(Y \cdot I)=\lim _{t \rightarrow 0}\langle Y(t)\rangle_{C_{t}}\langle 1\rangle_{C_{t}}=\frac{V}{2 \pi} .
$$

To calculate the left hand side we need the Schwinger representation

$$
\operatorname{Tr}\left[Y \cdot \frac{1}{K+\phi_{u}}\left(K+\phi_{u}\right)\right]=\int_{0}^{\infty} d t \operatorname{Tr}\left[Y \cdot e^{-t\left(K+\phi_{u}\right)}\left(K+\phi_{u}\right)\right]
$$

Making the replacement

$$
e^{-t\left(K+\phi_{u}\right)}\left(K+\phi_{u}\right) \rightarrow-\frac{d}{d t} e^{-t\left(K+\phi_{u}\right)}
$$

one obtains

$$
\operatorname{Tr}\left[Y \cdot \frac{1}{K+\phi_{u}}\left(K+\phi_{u}\right)\right]=g(0)-g(\infty)=\frac{V}{2 \pi}-g(\infty),
$$

which is different form (3.3) because $g(\infty)$ is nonvanishing. The latter relation is often written in a stronger form

$$
\int_{0}^{\infty} d t e^{-t\left(K+\phi_{u}\right)}\left(K+\phi_{u}\right)=1-\Omega_{u}^{\infty}, \quad \Omega_{u}^{\infty}=\lim _{\Lambda \rightarrow \infty} e^{-\Lambda\left(K+\phi_{u}\right)}
$$

This (strong) equality, however, has to be handled with great care. If the latter is taken literally, we could also write

$$
\frac{1}{K+\phi_{u}}=\int_{0}^{\infty} d t e^{-t\left(K+\phi_{u}\right)}+\frac{1}{K+\phi_{u}} \Omega_{u}^{\infty}
$$

instead of (3.1). This would imply that eq.(3.2) is not satisfied, and, consequently, the equation of motion is not satisfied by $\psi_{u}$. 


\section{An example from classical field theory}

The problem raised in the previous section is actually commonplace in the search of solutions in ordinary classical field theory and was solved long ago resorting to the theory of distributions, and tacitly incorporated in our common lore. Let us present one of many possible examples. We ask our patient reader to follow us through some elementary mathematics. We hope the example will help clarifying our line of thought in solving the puzzle raised in the previous section.

\subsection{Preliminaries}

In preparation for our $3 \mathrm{~d}$ example let us introduce some notation:

$$
\begin{aligned}
& x=r \sin \theta \cos \varphi, \quad y=r \sin \theta \sin \varphi, \quad z=r \cos \theta \\
& r=\sqrt{x^{2}+y^{2}+z^{2}}, \quad \theta=\arccos \frac{z}{r}, \quad \varphi=\arctan \frac{y}{x}
\end{aligned}
$$

and

$$
\Delta f=\frac{1}{r^{2}} \partial_{r}\left(r^{2} \partial_{r} f\right)+\frac{1}{r^{2} \sin \theta} \partial_{\theta}\left(\sin \theta \partial_{\theta} f\right)+\frac{1}{r^{2} \sin ^{2} \theta} \partial_{\varphi}^{2} f
$$

Distribution theory tells us that

$$
\Delta \frac{1}{r}=-4 \pi \delta(r)
$$

Now, let us consider the product $r \frac{1}{r}$. According to distribution theory (and to continuity) we should have

$$
r \frac{1}{r}=1
$$

In fact, using a test function $f(x, y, z)$, we have

$$
\begin{aligned}
& <r \frac{1}{r}, f>=\lim _{\varepsilon \rightarrow 0} \iiint_{r \geq \varepsilon} d x d y d z r \frac{1}{r} f(x, y, z) \\
= & \lim _{\varepsilon \rightarrow 0} \iiint_{r \geq \varepsilon} d x d y d z f(x, y, z)=\iiint d x d y d z f(x, y, z)=<1, f>
\end{aligned}
$$

Therefore $r \frac{1}{r}$, as a distribution, is 1 . In view of the previous section one might decide to use a Schwinger representation

$$
r \frac{1}{r} \longrightarrow \int_{0}^{\infty} d t r e^{-t r}=-\int_{0}^{\infty} d t \frac{\partial}{\partial t} e^{-t r}=1-\lim _{t \rightarrow \infty} e^{-t r} \equiv 1-\Omega(r)
$$

Consequently $\frac{1}{r}$ is represented by

$$
\frac{1}{r} \longrightarrow \int_{0}^{\infty} d t e^{-t r}+\Pi(r), \quad \Pi(r)=\frac{\Omega(r)}{r}
$$

Let us elaborate a bit on this in order to prepare the ground for our example. From (4.4) it is clear that $\Omega(r)$ has support at $r=0$, therefore it must be a delta-function-like object. Using the definition of delta function as a limit

$$
\lim _{t \rightarrow \infty} \sqrt{\frac{t}{\pi}} e^{-t x^{2}}=\delta(x)
$$


we can set

$$
\Omega(r)=2 \lim _{t \rightarrow \infty} \sqrt{\frac{\pi r}{t}} \delta(r)
$$

This is the only way to bring $\Omega(r)$ in the world of well-defined objects. (4.6) is obviously an identically vanishing distribution. But, of course, if we integrate it over something which is not a test function, we may get a nonvanishing result. For later use let us define also

$$
\Pi(r)=2 \lim _{t \rightarrow \infty} \sqrt{\frac{\pi}{r t}} \delta(r), \quad \Xi(r)=2 \lim _{t \rightarrow \infty} \sqrt{\pi r t} \delta(r)
$$

Let us make a comparison between (4.3) and (4.4). An explicit calculation yields

$$
\Delta\left(r \frac{1}{r}\right)=-4 \pi r \delta(r)+\frac{2}{r^{2}}-\frac{2}{r^{2}}=0
$$

as a distribution. This is a result of (4.2), of $\Delta r=\frac{2}{r}$ and of

$$
\sum_{i=1}^{3} \partial_{x_{i}} \frac{1}{r} \partial_{x_{i}} r=-\frac{1}{r^{2}}
$$

This last calculation is straightforward for $r \neq 0$, but at the origin one must be careful and use distribution theory: for a test function $f(x, y, z)$ we can write, for example,

$$
\begin{aligned}
& \iiint d x d y d z \partial_{x}\left(\frac{1}{r}\right) f(x, y, z)=-\lim _{\varepsilon \rightarrow 0} \iiint_{r \geq \varepsilon} d x d y d z \sin \theta \cos \varphi \frac{1}{r^{2}} f(x, y, z) \\
& -\lim _{\varepsilon \rightarrow 0} \iiint_{r \geq \varepsilon} d r d \theta d \varphi \sin ^{2} \theta \cos \varphi f(r, \theta, \varphi)=-\iiint d r d \theta d \varphi \sin ^{2} \theta \cos \varphi f(r, \theta, \varphi)
\end{aligned}
$$

which means that the distributional derivative $\partial_{x} \frac{1}{r}$ coincides with the ordinary derivative (there is no extra contribution from $r=0$ ).

On the other hand, using the representation (4.4), we have

$$
\Delta\left(r \frac{1}{r}\right)=-\Delta \Omega(r)
$$

The RHS is formally nonvanishing since $\Delta(\sqrt{r} \delta(r))=\frac{3}{4} r^{-\frac{3}{2}} \delta(r)+3 r^{-\frac{1}{2}} \boldsymbol{\delta}^{\prime}(r)+r^{\frac{1}{2}} \boldsymbol{\delta}^{\prime \prime}(r)$. However, remembering that the volume element contains a factor of $r^{2}, \Delta \Omega(r)$ is in fact the 0 distribution. This is consistent with (4.8). But if we do not correctly apply the rules of distribution theory the RHS of (4.10) may seem to be nonvanishing (although ambiguous). This may happen, for instance, if we integrate such term multiplied by a function that is more singular than $\frac{1}{\sqrt{r}}$ for $r \approx 0$. The trouble is that such a function is not a test function.

\subsection{The Schwarzschild black hole 'non-solution'}

Let us check on an example that the (wrong) use of (4.10) leads to wrong results. To this end we consider the Schwarzschild solution in gravity. The Schwarzschild geometry is a solution to the Einstein equation in vacuum: $R_{\mu \nu}=0$. 
Let us consider the ordinary approach. The Schwarzschild metric has the form

$$
d s^{2}=-f(r) d t^{2}+\frac{d r^{2}}{f(r)}+r^{2}\left(d \theta^{2}+\sin ^{2} \theta d \varphi^{2}\right)
$$

so that we have

$$
g_{00}=-f(r), \quad g_{r r}=\frac{1}{f(r)}, \quad g_{\theta \theta}=r^{2}, \quad g_{\varphi \varphi}=r^{2} \sin ^{2} \theta
$$

where, for simplicity, we take $f(r)=1-\frac{2 M}{r}$. The Christoffel symbols are

$$
\Gamma_{0 r}^{0}=\frac{f^{\prime}}{2 f}=-\Gamma_{r r}^{r}, \quad \Gamma_{00}^{r}=\frac{f f^{\prime}}{2}, \quad \Gamma_{\theta \theta}^{r}=-r f, \quad \Gamma_{\varphi \varphi}^{r}=-r f \sin ^{2} \theta
$$

where $f^{\prime}(r)=\frac{d f(r)}{d r}$. There are other (completely angular) nonvanishing symbols but we will not need them. As a consequence in particular we have

$$
R_{0 r 0 r}=\frac{f^{\prime \prime}}{2}, \quad R_{0 \theta 0 \theta}=\frac{1}{2} r f f^{\prime}, \quad R_{0 \varphi 0 \varphi}=\frac{1}{2} r f f^{\prime} \sin ^{2} \theta
$$

At this point it is easy to prove, for instance, that

$$
R_{00}=g^{r r} R_{0 r 0 r}+g^{\theta \theta} R_{0 \theta 0 \theta}+g^{\varphi \varphi} R_{0 \varphi 0 \varphi}=0
$$

so that the eom is satisfied (for the 00 case).

In all the above, $\frac{1}{f}$ is singular at the horizon $r=2 M$, so that one component of the metric is singular. However the Riemann tensor is not singular (and the energy is finite). In the intermediate passages we have to manipulate $\frac{1}{f}$ or derivative thereof. This is singular, but interpreting it and carrying out all the operations in the framework of distribution theory all the singularities can be treated correctly and the final result is regular.

Now let us see what happens instead when we use improperly the Schwinger representation for $\frac{1}{f}$. To this end let us call

$$
S\left(\frac{1}{f}\right)=\int_{0}^{\infty} d t e^{-t f}
$$

the Schwinger representation of $\frac{1}{f}$. We have

$$
\begin{aligned}
& f S\left(\frac{1}{f}\right)=1-\Omega(f), \quad \frac{1}{f}=S\left(\frac{1}{f}\right)+\Pi(f) \\
& f \Pi(f)=\Omega(f), \quad f \partial_{r} S\left(\frac{1}{f}\right)=f^{\prime} \Xi(f)-f^{\prime} S\left(\frac{1}{f}\right)
\end{aligned}
$$

The last one follows from

$$
\begin{aligned}
f \partial_{r} S\left(\frac{1}{f}\right) & =-\int_{0}^{\infty} d t t f^{\prime} f e^{-t f}=f^{\prime} \int_{0}^{\infty} d t t \frac{d}{d t} e^{-t f} \\
& =f^{\prime} \int_{0}^{\infty} d t \frac{d}{d t}\left(t e^{-t f}\right)-f^{\prime} \int_{0}^{\infty} d t e^{-t f}=2 f^{\prime} \lim _{t \rightarrow \infty} \sqrt{\pi t f} \delta(f)-f^{\prime} S\left(\frac{1}{f}\right)
\end{aligned}
$$


Of the relevant Christoffel symbols $\Gamma_{0 r}^{0}, \Gamma_{r r}^{r}$ are singular, while the others are regular. More precisely we have

$$
\Gamma_{0 r}^{0}=\frac{f^{\prime}}{2}\left(S\left(\frac{1}{f}\right)+\Pi(f)\right), \quad \Gamma_{r r}^{r}=\frac{f^{\prime}}{2}\left(\Xi(f)-S\left(\frac{1}{f}\right)\right)
$$

Repeating the calculation with these inputs we get

$$
\begin{aligned}
R_{0 r 0 r} & =\frac{f^{\prime \prime}}{2}+\frac{f f^{\prime}}{2} \Pi^{\prime}(f)+\frac{f^{\prime} f^{\prime}}{4}\left(\Xi(f)+\Omega(f) S\left(\frac{1}{f}\right)+\Omega(f) \Pi(f)\right) \\
R_{0 \theta 0 \theta} & =\frac{1}{2} r f f^{\prime}, \quad R_{0 \varphi 0 \varphi}=\frac{1}{2} r f f^{\prime} \sin ^{2} \theta
\end{aligned}
$$

Therefore

$$
\begin{aligned}
R_{00}= & \frac{f f^{\prime \prime}}{2}+\frac{f f^{\prime}}{r} \\
& +\frac{f f^{\prime} f^{\prime}}{4} \Xi(f)+\frac{f^{\prime} f^{\prime}}{4} \Omega(f)+\frac{f^{2} f^{\prime}}{2} \Pi^{\prime}(f)
\end{aligned}
$$

The first line is the usual (vanishing) result, the second line represents the violation to the eom. Notice that in the framework of distribution theory the second line vanishes, but if one takes the previous algebraic manipulations literally one might conclude that Schwarzschild's is not a solution of Einstein gravity. In particular if we integrate the second line over a non-test function we may get something different from 0 . This is no accident: these terms are intrinsically ambiguous, as is evident if one tries to define them carefully. Terms such as those in the second line of (4.20) are inevitably ambiguous when considered outside the framework of distribution theory. We will refer to them as spurious terms.

This is an example of what we run into when we abandon the principle of continuity (or analyticity) according to which the statement: $r \frac{1}{r}=1$ everywhere, is the correct thing. This principle has been incorporated into the theory of distributions, which, in this way, has eliminated all the above ambiguities (a distribution is defined via Riemann integrals, which in turn are defined by means of continuous limiting processes, so they automatically incorporate the principle of continuity). But if we abandon this principle we end up in a jungle of contradictions.

\section{Continuity and the Schwinger representation}

The previous example may sound somewhat exotic, but in every respect it is a paradigm of the problem introduced in section 3 . Let us now return to it.

In our approach in $[2,4]$ we have always been guided by what we have called above the principle of continuity. On the basis of this principle (3.1), as opposed to (3.8), is the correct relation. Let us summarize how we discussed this issue in Appendix D of [2]. We start from the observation that $K+\phi_{u}$ is a vector in an infinite dimensional space: $K+\phi_{u}=\left(K_{1}^{L}+\phi_{u}\left(\frac{1}{2}\right)\right)|I\rangle$, where $|I\rangle$ is the identity string field (and we remark that in our applications $\phi_{u}(\tilde{z})$ is always inserted in the left part of the string). Therefore the inverse of $K+\phi_{u}$ can also be obtained via the inverse of the operator $\mathscr{K}_{u} \equiv K_{1}^{L}+\phi_{u}\left(\frac{1}{2}\right)$.

The operator $\mathscr{K}_{u}$ is self-adjoint. Therefore its spectrum lies on the real axis. To know more about it we would need a spectral analysis of $\mathscr{K}_{u}$, similar to what has been done for the operator 
$K_{1}^{L}$ in $[17,19,20,21]$. The spectrum of the latter is the entire real axis. The spectrum of $\mathscr{K}_{u}$ is of course expected to be different, but we know on a general ground that it lies on the real axis. We can therefore define the resolvent of $\mathscr{K}_{u}, R\left(\kappa, \mathscr{K}_{u}\right)$, which is by definition the inverse of $\kappa-\mathscr{K}_{u}$ ( $\kappa$ being a complex parameter). The resolvent is well defined (at least) for any non-real $\kappa$. We do not know what type of eigenvalue the $\kappa=0$ one is: discrete, continuous or residual. However, since $R\left(\kappa, \mathscr{K}_{u}\right)\left(\kappa-\mathscr{K}_{u}\right)=1$ is true for any $\kappa$ outside the real axis, we can hold it valid also in the limit $\kappa \rightarrow 0$ by continuity. Therefore we conclude that, on the basis of the (healthy) principle of continuity, (3.1) is the correct relation, much as $r \frac{1}{r}=1$ was held true everywhere in the previous section.

The obvious difference between the two cases is that in the previous section's case we were talking about the inverse of a position $r$, while in this section we are talking about the inverse of a string field $K+\phi_{u}$. We remark however that this is the natural correspondence when we pass from classical gravity (classical field theory) to SFT: the role of positions in the former is played by string configurations in the latter.

One may object at this point that, true, since (3.1) is correct, the SFT equation of motion is satisfied by our solution, but in order to compute its energy we need the Schwinger representation of the inverse of $K+\phi_{u}$. Given the ambiguity of the latter (see (3.7) and (3.8)) brought about by the term $\Omega_{u}^{\infty}$, one may wonder whether the computation of the energy may be altered by the presence of such terms.

On the basis of the analogy with the previous section we are led to conclude that such ambiguous terms have to be identified as spurious ones. We have argued above that a good hygienic rule is to drop them. Keeping them may be useless in the best case and misleading in the worst. In any case we would like to modestly remark that, should we find that the Schwinger representation is defective in calculating the energy, the most logical course would be to correct it, not to blame the solution for not satisfying the equation of motion. Fortunately, anyhow, this will not be necessary. The Schwinger representation perfectly does its job, provided it is handled with care. In fact we will show that spurious terms yield vanishing contributions if inserted in converging integrals, while they may give nonvanishing (but ambiguous) contributions only if they appear in divergent integrands.

\section{Concerning the identity $\frac{1}{K+\phi_{u}}\left(K+\phi_{u}\right)=I$}

Let us return to section 2 and eqs.(3.2), (3.1) and (3.8). Applying our new representation we get

$$
\begin{aligned}
\frac{1}{K+\phi_{u}}\left(K+\phi_{u}\right) & =\sum_{n=0}^{\infty} \frac{(-\varepsilon)^{n}}{n !} \partial_{\varepsilon}^{n} \frac{1}{K+\phi_{u}+\varepsilon}\left(K+\phi_{u}\right) \\
& =e^{-\varepsilon \partial_{\varepsilon}}\left(1-\frac{\varepsilon}{K+\phi_{u}+\varepsilon}\right) \\
& =1-e^{-\varepsilon \partial_{\varepsilon}} \frac{\varepsilon}{K+\phi_{u}+\varepsilon}
\end{aligned}
$$

The expression $e^{-\varepsilon \partial_{\varepsilon}} \frac{\varepsilon}{K+\phi_{u}+\varepsilon}=\lim _{\varepsilon \rightarrow 0} \frac{\varepsilon}{K+\phi_{u}+\varepsilon}$ is a more appropriate way to write $\Omega_{u}^{\infty}$ (it is extremely helpful to keep in mind the analogy with $\Omega(r)$ in sec. 3). It is of course formally vanishing, 
but to make any sense of such an expression one has to evaluate it in correlators. For instance, taking the trace, as in section 2, we are led to evaluate

$$
\operatorname{Tr}\left[\frac{\varepsilon}{K+\phi_{u}+\varepsilon}\right]=\varepsilon \int_{0}^{\infty} d t e^{-\varepsilon t} g(u t)
$$

Since, once again, $g(\infty)=1$, the limit $\varepsilon \rightarrow 0$ is not continuous, and this depends on the fact that, as we have seen many times, the integral in the RHS of (6.2) is (linearly) divergent when the factor $e^{-\varepsilon t}$ is replaced by 1 . As a consequence the shift operator $e^{-\varepsilon \partial_{\varepsilon}}$ cannot be applied in a consistent way in (6.2). In fact it is not clear what value one should assign to the expression

$$
e^{-\varepsilon \partial_{\varepsilon}}\left(\varepsilon \int_{0}^{\infty} d t e^{-\varepsilon t} g(u t)\right)
$$

depending on whether we integrate first or apply first the operation $e^{-\varepsilon \partial_{\varepsilon}} \varepsilon$ to the integrand.

On the other hand, if (6.1) is inserted in a correlator (like the energy one) where the integrand without the exponential factor decreases fast enough, then the result of the application of $e^{-\varepsilon \partial_{\varepsilon}}$ to $\frac{\varepsilon}{K+\phi_{u}+\varepsilon}$ is unambiguously 0 . This can be seen by considering for instance the following contraction

$$
\begin{aligned}
& \operatorname{Tr}\left[\partial^{2} c e^{-(K+\phi)} e^{-\varepsilon \partial_{\varepsilon}}\left(\frac{\varepsilon}{\left(K+\phi_{u}+\varepsilon\right)}\left(\phi_{u}-\delta \phi_{u}\right) c \partial c\right)\right] \\
& =e^{-\varepsilon \partial_{\varepsilon}} \varepsilon \int_{0}^{\infty} d t e^{-\varepsilon t} \operatorname{Tr}\left[\left(\phi_{u}-\delta \phi_{u}\right) e^{-(t+1)(K+\phi)}\right]\left\langle\partial^{2} c(t+1) c \partial c(0)\right\rangle_{C_{t+1}} \\
& =e^{-\varepsilon \partial_{\varepsilon}} \varepsilon \int_{0}^{\infty} d t e^{-\varepsilon t}\left\langle\left(\phi_{u}(0)-\delta \phi_{u}(0)\right) e^{-\int_{0}^{t+1} d s \phi(s)}\right\rangle_{C_{t+1}}\left\langle\partial^{2} c(t+1) c \partial c(0)\right\rangle_{C_{t+1}} \\
& =-e^{-\varepsilon \partial_{\varepsilon}} \varepsilon \int_{0}^{\infty} d t e^{-\varepsilon t} G(t) \frac{u}{t+1} \partial_{u} g(u(t+1))=2 e^{-\varepsilon \partial_{\varepsilon}} \varepsilon \int_{0}^{\infty} d t e^{-\varepsilon t} \frac{u}{t+1} \partial_{u} g(u(t+1))
\end{aligned}
$$

where the ghost contribution is given by

$$
G(t)=\left\langle\partial^{2} c(t+1)(c \partial c)(0)\right\rangle_{C_{t+1}}=-2
$$

Now we can write eq.(6.4) as

$$
\begin{aligned}
2\left(e^{-\varepsilon \partial_{\varepsilon}} \varepsilon\right) e^{-\varepsilon \partial_{\varepsilon}} & \int_{0}^{\infty} d t e^{-\varepsilon t} \frac{u}{t+1} \partial_{u} g(u(t+1)) \\
& =2\left(e^{-\varepsilon \partial_{\varepsilon}} \mathcal{\varepsilon}\right) \int_{0}^{\infty} d t \frac{u}{t+1} \partial_{u} g(u(t+1))=0 .
\end{aligned}
$$

We note that this last result does not need any UV subtraction.

\subsection{How to compute correlators with spurious terms}

After these long preliminaries let us come to the would-be violation of the equation of motion due to the second term in the RHS of (6.1), pointed out in [3]. To this end we rewrite

$$
\psi_{u} \rightarrow \psi_{u, \varepsilon}=c \phi_{u}-e^{-\varepsilon \partial_{\varepsilon}} \frac{1}{\left(K+\phi_{u}+\varepsilon\right)}\left(\phi_{u}-\delta \phi_{u}\right) B c \partial c
$$

and apply $Q$ to it. Using in particular

$$
Q\left(e^{-\varepsilon \partial_{\varepsilon}} \frac{1}{\left(K+\phi_{u}+\varepsilon\right)}\right)=-e^{-\varepsilon \partial_{\varepsilon}} \frac{1}{\left(K+\phi_{u}+\varepsilon\right)}\left(Q \phi_{u}\right) \frac{1}{\left(K+\phi_{u}+\varepsilon\right)}
$$


and proceeding as in section 3.2 of [1], we find

$$
\begin{aligned}
& Q \psi_{u, \varepsilon}=Q\left(c \phi_{u}-e^{-\varepsilon \partial_{\varepsilon}} \frac{1}{\left(K+\phi_{u}+\varepsilon\right)}\left(\phi_{u}-\delta \phi_{u}\right) B c \partial c\right) \\
& =e^{-\varepsilon \partial_{\varepsilon}}\left[1+\frac{1}{\left(K+\phi_{u}+\varepsilon\right)}\left(c \partial \phi_{u}+\partial c \delta \phi_{u}\right) \frac{1}{\left(K+\phi_{u}+\varepsilon\right)} B-\frac{1}{\left(K+\phi_{u}+\varepsilon\right)} K\right]\left(\phi_{u}-\delta \phi_{u}\right) c \partial c \\
& =e^{-\varepsilon \partial_{\varepsilon}}\left[\left(c \phi_{u}-\frac{1}{\left(K+\phi_{u}+\varepsilon\right)}\left(\phi_{u}-\delta \phi_{u}\right) \partial c\right) \frac{1}{\left(K+\phi_{u}+\varepsilon\right)}+\frac{\varepsilon}{\left(K+\phi_{u}+\varepsilon\right)} c\right]\left(\phi_{u}-\delta \phi_{u}\right) B c \partial c \\
& =-\psi_{u, \varepsilon} \psi_{u, \varepsilon}+e^{-\varepsilon \partial_{\varepsilon}}\left(\frac{\varepsilon}{\left(K+\phi_{u}+\varepsilon\right)}\left(\phi_{u}-\delta \phi_{u}\right) c \partial c\right)
\end{aligned}
$$

In a regular setting, that is when inserted in a correlator regular in $\varepsilon$, this boils down to the usual eom $Q \psi_{u}=-\psi_{u} \psi_{u}$, and in particular the second piece in the RHS of the last line vanishes. Let us see what happens if we, nevertheless, insist in keeping (6.8) in the expression of the energy. We have

$$
\begin{aligned}
-\left\langle\psi_{u} Q \psi_{u}\right\rangle & \rightarrow-\left\langle\psi_{u, \varepsilon} Q \psi_{u, \varepsilon}\right\rangle \\
& =\left\langle\psi_{u, \varepsilon} \psi_{u, \varepsilon} \psi_{u, \varepsilon}\right\rangle+\left\langle\psi_{u, \varepsilon} e^{-\varepsilon \partial_{\varepsilon}}\left(\frac{\varepsilon}{\left(K+\phi_{u}+\varepsilon\right)}\left(\phi_{u}-\delta \phi_{u}\right) c \partial c\right)\right\rangle
\end{aligned}
$$

The second term in the RHS equals

$$
e^{-\varepsilon \partial_{\varepsilon}}\left\langle\frac{1}{\left(K+\phi_{u}+\varepsilon\right)}\left(\phi_{u}-\delta \phi_{u}\right) B c \partial c \frac{\varepsilon}{\left(K+\phi_{u}+\varepsilon\right)}\left(\phi_{u}-\delta \phi_{u}\right) c \partial c\right\rangle
$$

With the usual procedure we can write this as $\left(T=t_{1}+t_{2}\right)$

$$
e^{-\varepsilon \partial_{\varepsilon}}\left(\varepsilon \int_{0}^{\infty} d t_{1} d t_{2} e^{-\varepsilon T} \mathscr{G}\left(t_{1}, t_{2}\right) u^{2} g(u T)\left\{\left(-\frac{\partial_{u T} g(u T)}{g(u T)}\right)^{2}+2 G_{2 u T}^{2}\left(\frac{2 \pi t_{1}}{T}\right)\right\}\right),
$$

where the ghost part is given by

$$
\mathscr{G}\left(t_{1}, t_{2}\right)=\left\langle(B c \partial c)\left(t_{1}\right)(c \partial c)(0)\right\rangle_{C_{T}}=\frac{t_{1}}{\pi} \sin \left(\frac{2 \pi t_{1}}{T}\right)-\frac{2 T}{\pi^{2}} \sin ^{2}\left(\frac{\pi t_{1}}{T}\right) .
$$

Let us show now that $(6.11)$ reduces to the form

$$
e^{-\varepsilon \partial_{\varepsilon}}\left(\varepsilon \int_{0}^{\infty} d s e^{-\varepsilon s} \mathcal{F}(s)\right)
$$

where $\mathcal{F}(s) \rightarrow$ const for large $s$ and the integral is UV finite.

Denoting $x=\frac{t_{1}}{T}$, Eq.(6.12) can be rewritten as

$$
e^{-\tilde{\eta} \partial_{\tilde{\eta}}} \tilde{\eta} \int_{0}^{\infty} d s s^{2} \int_{0}^{1} d x \mathscr{E}(x) e^{-\tilde{\eta} s} \mathrm{~g}(s)\left\{\left(-\frac{\partial_{s} \mathrm{~g}(s)}{\mathrm{g}(s)}\right)^{2}+\frac{1}{2} G_{s}^{2}(2 \pi x)\right\},
$$

where $\tilde{\eta}=\frac{\varepsilon}{2 u}$ and

$$
\mathscr{E}(x)=\langle(B c \partial c)(x)(c \partial c)(0)\rangle_{C_{1}}=\frac{-1+\cos (2 \pi x)+\pi x \sin (2 \pi x)}{\pi^{2}}
$$


Since $\int_{0}^{1} d x \mathscr{E}(x)=-\frac{3}{2 \pi^{2}}$, the term with no $G_{S}$ is given by

$$
-\frac{3}{2 \pi^{2}} \tilde{\eta} \int_{0}^{\infty} d s s^{2} e^{-\tilde{\eta} s} \mathrm{~g}(s)\left(-\frac{\partial_{s} \mathrm{~g}(s)}{\mathrm{g}(s)}\right)^{2}
$$

As $g(s) \approx \frac{1}{\sqrt{s}}$ in the UV we are in the case of eq.(8.13) of [2] and so the UV contribution vanishes for $\tilde{\eta} \rightarrow 0$. In the IR we are in the case of eq.(8.17) of [2] and so the IR contribution vanishes too. It can be easily proven that

$$
\begin{array}{r}
3 \int_{0}^{1} d x \mathscr{E}(x) G_{s}^{2}(2 \pi x)=\frac{4}{\pi} \int_{0}^{1} d y \int_{0}^{y} d x \sin \pi x \sin \pi y \sin \pi(x-y) \\
\cdot\left(G_{s}^{2}(2 \pi x)+G_{s}^{2}(2 \pi(x-y))+G_{s}^{2}(2 \pi y)\right)
\end{array}
$$

where the expression in the RHS is the same as eq.(3.7) of [2]. Therefore we have

$$
\begin{aligned}
& e^{-\tilde{\eta} \partial_{\tilde{\eta}}}\left(\frac{1}{2} \tilde{\eta} \int_{0}^{\infty} d s s^{2} e^{-\tilde{\eta} s} \mathrm{~g}(s) \int_{0}^{1} d x \mathscr{E}(x, 0) G_{s}^{2}(2 \pi x)\right) \\
&=e^{-\tilde{\eta} \partial_{\tilde{\eta}}}\left(\frac{1}{6} \tilde{\eta} \int_{0}^{\infty} d s s^{2} e^{-\tilde{\eta} s} \mathrm{~g}(s) \frac{4}{\pi} \int_{0}^{1} d y \int_{0}^{y} d x \sin \pi x \sin \pi y \sin \pi(x-y)\right. \\
&\left.\cdot\left(G_{s}^{2}(2 \pi x)+G_{s}^{2}(2 \pi(x-y))+G_{s}^{2}(2 \pi y)\right)\right)
\end{aligned}
$$

We can now avail ourselves of the results in [2]. The integration over $x$ and $y$ leads to an integrand in $s$ that behaves like a constant for large $s$, if one abstracts from the factor $e^{-\tilde{\eta} s}$. Thus we have obtained (6.13). Under these conditions the limit for $\tilde{\eta} \rightarrow 0$ of the $s$ integral is discontinuous and we are not allowed to exchange $e^{-\tilde{\eta} \partial_{\tilde{\eta}}}$ with the integration. We do not know what value should be assigned to (6.13). As a consequence the additional piece in RHS of (6.9) cannot be assigned an unambiguous value without an ad hoc prescription.

With an $a d$ hoc prescription we can still obtain a finite result. If, for instance, we first multiply $\tilde{\eta}$ by the result of the integration and subsequently apply $e^{-\tilde{\eta} \partial_{\tilde{\eta}}}$ we obtain $-2 \beta$, where $\beta$ is the number introduced in [2], see also sec.2 above. This result is the same as the one obtained by [3]. But one should not forget that it is prescription-dependent. We remark, in addition, that the term (6.18) appears in the RHS of eq.(6.9) together with $\left\langle\psi_{u, \varepsilon} \psi_{u, \varepsilon} \psi_{u, \varepsilon}\right\rangle$. The latter is a UV divergent term (in [4] it is even gauge-dependent) and needs a UV subtraction, which, we recall, carries some arbitrariness into the problem. For instance, one could choose the UV subtraction in such a way as to kill the contribution of $-2 \beta$ altogether and there would be no violation of the EOM. Therefore it is not even clear what the would-be violation of the EOM means.

The ambiguity intrinsic in this problem reminds us of the discussion after eq.(4.10) in sec. 3. There, by integrating a vanishing distribution over a non test function, we could obtain a nonvanishing result. This is no accident. The nonvanishing of the second term in the RHS of (6.9) is analogous. The string field $e^{-\varepsilon \partial_{\varepsilon}} \frac{\varepsilon}{K+\phi_{u}+\varepsilon}\left(\phi_{u}-\delta \phi_{u}\right)$ plays the role of the vanishing distribution and $\psi_{u, \varepsilon}$ the role of the singular test function. The only difference here is that the singularity comes from the IR, because of the inversion of roles introduced by the Schwinger representation. In this regard we can be more precise. If we strip (6.10) of the $\varepsilon$ factor in the numerator, what remains represents the string field $\frac{1}{K+\phi_{u}+\varepsilon}\left(\phi_{u}-\delta \phi_{u}\right)$ contracted with itself, which can be interpreted as the 'norm' square of this string field, in the limit $\varepsilon \rightarrow 0$. Well, the above results tell us that this 'norm' 
is infinite. It is this infinity that multiplied by the stripped factor $\varepsilon$ allows us to obtain the above finite result. This clearly confirms the singular nature of $\psi_{u, \varepsilon}$ as a test state.

It is instead possible to derive a prescription-independent (and subtraction-independent) result, even taking into account the spurious term, provided one proceeds in another way. Let us rewrite $\Phi_{0}^{\varepsilon}$, eq. (1.24), using the new representation: $\Phi(\varepsilon, \varepsilon)=\psi_{u, \varepsilon}-\psi_{u}^{\varepsilon}$, where $\psi_{u}^{\varepsilon}$, in the $\varepsilon \rightarrow 0$ limit, is the tachyon vacuum solution defined in [2]. We get

$$
\begin{aligned}
Q \psi_{u, \varepsilon} & =-\psi_{u, \varepsilon} \psi_{u, \varepsilon}+e^{-\varepsilon \partial_{\varepsilon}}\left(\frac{\varepsilon}{\left(K+\phi_{u}+\varepsilon\right)}\left(\phi_{u}-\delta \phi_{u}\right) c \partial c\right) \\
Q \psi_{u}^{\varepsilon} & =-\psi_{u}^{\varepsilon} \psi_{u}^{\varepsilon} \\
\mathscr{Q} \Phi(\varepsilon, \varepsilon) & =-\Phi(\varepsilon, \varepsilon) \Phi(\varepsilon, \varepsilon)+e^{-\varepsilon \partial_{\varepsilon}}\left(\frac{\varepsilon}{\left(K+\phi_{u}+\varepsilon\right)}\left(\phi_{u}-\delta \phi_{u}\right) c \partial c\right)
\end{aligned}
$$

where $\mathscr{Q} \Phi=Q \Phi+\psi_{u}^{\varepsilon} \Phi+\Phi \psi_{u}^{\varepsilon}$. Moreover

$$
\begin{aligned}
-\langle\Phi(\varepsilon, \varepsilon) \mathscr{Q} \Phi(\varepsilon, \varepsilon)\rangle= & \langle\Phi(\varepsilon, \varepsilon) \Phi(\varepsilon, \varepsilon) \Phi(\varepsilon, \varepsilon)\rangle \\
& +\left\langle\Phi(\varepsilon, \varepsilon) e^{-\varepsilon \partial_{\varepsilon}}\left(\frac{\varepsilon}{\left(K+\phi_{u}+\varepsilon\right)}\left(\phi_{u}-\delta \phi_{u}\right) c \partial c\right)\right\rangle
\end{aligned}
$$

If we use the just defined representation, the second term in the RHS equals

$$
\begin{aligned}
& e^{-\varepsilon \partial_{\varepsilon}}\left\langle\psi_{u, \varepsilon}\left(\frac{\varepsilon}{\left(K+\phi_{u}+\varepsilon\right)}\left(\phi_{u}-\delta \phi_{u}\right) c \partial c\right)\right\rangle-e^{-\varepsilon \partial_{\varepsilon}}\left\langle\psi_{u}^{\varepsilon}\left(\frac{\varepsilon}{\left(K+\phi_{u}+\varepsilon\right)}\left(\phi_{u}-\delta \phi_{u}\right) c \partial c\right)\right\rangle \\
= & -2 \beta-e^{-\varepsilon \partial_{\varepsilon}}\left\langle\frac{1}{\left(K+\phi_{u}+\varepsilon\right)}\left(\phi_{u}+\varepsilon-\delta \phi_{u}\right) B c \partial c \frac{\varepsilon}{\left(K+\phi_{u}+\varepsilon\right)}\left(\phi_{u}-\delta \phi_{u}\right) c \partial c\right\rangle \\
= & -2 \beta-e^{-\varepsilon \partial_{\varepsilon}}\left\langle\frac{1}{\left(K+\phi_{u}+\varepsilon\right)}\left(\phi_{u}-\delta \phi_{u}\right) B c \partial c \frac{\varepsilon}{\left(K+\phi_{u}+\varepsilon\right)}\left(\phi_{u}-\delta \phi_{u}\right) c \partial c\right\rangle \\
& -e^{-\varepsilon \partial_{\varepsilon}}\left\langle\frac{\varepsilon}{\left(K+\phi_{u}+\varepsilon\right)} B c \partial c \frac{\varepsilon}{\left(K+\phi_{u}+\varepsilon\right)}\left(\phi_{u}-\delta \phi_{u}\right) c \partial c\right\rangle
\end{aligned}
$$

In (6.21) there is no need of UV subtractions. The last two terms in the RHS equal, respectively,

$$
\begin{aligned}
& e^{-\varepsilon \partial_{\varepsilon}}\left\langle\frac{1}{\left(K+\phi_{u}+\varepsilon\right)}\left(\phi_{u}-\delta \phi_{u}\right) B c \partial c \frac{\varepsilon}{\left(K+\phi_{u}+\varepsilon\right)}\left(\phi_{u}-\delta \phi_{u}\right) c \partial c\right\rangle \\
= & e^{-\varepsilon \partial_{\varepsilon}}\left(\varepsilon \int_{0}^{\infty} d t_{1} d t_{2} e^{-\varepsilon t_{2}-\varepsilon t_{1}} \mathscr{G}\left(t_{1}, t_{2}\right) u^{2} g(u T)\left\{\left(-\frac{\partial_{u T} g(u T)}{g(u T)}\right)^{2}+2 G_{2 u T}^{2}\left(\frac{2 \pi t_{1}}{T}\right)\right\}\right) \\
= & e^{-\tilde{\eta} \partial_{\tilde{\eta}}}\left(\tilde{\eta} \int_{0}^{\infty} d s s^{2} \int_{0}^{1} d x \mathscr{E}(x) e^{-T(\varepsilon(1-x)+\varepsilon x)} \mathrm{g}(s)\left\{\left(-\frac{\partial_{s} \mathrm{~g}(s)}{\mathrm{g}(s)}\right)^{2}+\frac{1}{2} G_{s}^{2}(2 \pi x)\right\}\right) \\
= & e^{-\tilde{\eta} \partial_{\tilde{\eta}}}\left(\tilde{\eta} \int_{0}^{\infty} d s s^{2} \int_{0}^{1} d x e^{-\tilde{\eta} s} \mathscr{E}(1-x) e^{s \frac{\varepsilon-\varepsilon}{2 u} x} \mathrm{~g}(s)\left\{\left(-\frac{\partial_{s} \mathrm{~g}(s)}{\mathrm{g}(s)}\right)^{2}+\frac{1}{2} G_{s}^{2}(2 \pi x)\right\}\right)
\end{aligned}
$$

and

$$
\begin{aligned}
& e^{-\varepsilon \partial_{\varepsilon}}\left\langle\frac{\varepsilon}{\left(K+\phi_{u}+\varepsilon\right)} B c \partial c \frac{\varepsilon}{\left(K+\phi_{u}+\varepsilon\right)}\left(\phi_{u}-\delta \phi_{u}\right) c \partial c\right\rangle \\
= & e^{-\varepsilon \partial_{\varepsilon}}\left(\varepsilon \varepsilon \int_{0}^{\infty} d t_{1} d t_{2} e^{-\varepsilon t_{2}-\varepsilon t_{1}} \mathscr{G}\left(t_{1}, t_{2}\right) \frac{u}{t_{1}+t_{2}} \partial_{u} g(u T)\right) \\
= & e^{-\varepsilon \partial_{\varepsilon}}\left(\varepsilon \varepsilon \int_{0}^{\infty} d T T \int_{0}^{1} d x e^{-T(\varepsilon(1-x)+\varepsilon x)} \mathscr{E}(x) u \partial_{u} g(u T)\right) \\
= & e^{-\tilde{\eta} \partial_{\tilde{\eta}}}\left(\tilde{\eta} \frac{\varepsilon}{2 u} \int_{0}^{\infty} d s s^{2} e^{-\tilde{\eta} s} \int_{0}^{1} d x \mathscr{E}(1-x) e^{s \frac{\varepsilon-\varepsilon}{2 u} x} \partial_{s} \mathrm{~g}(s)\right)
\end{aligned}
$$


As we have learnt in section 2 these quantities must be evaluated in the limit $\varepsilon \rightarrow 0$. We are by now very familiar with this type of integrals and can easily come to the conclusion that both angular integrations are finite even without the $e^{\frac{\varepsilon-\varepsilon}{2 u} x}$ factors so that in the limit $\varepsilon, \varepsilon \rightarrow 0$ the integration is continuous in $\varepsilon, \varepsilon$ and such factors can be dropped. Thus, using always the same representation, the former integral is just $-2 \beta$. The latter is the same as eq.(4.43) of [3]. It is convergent both in the UV and the IR.

So we find

$$
\lim _{\varepsilon \rightarrow 0}\left\langle\Phi(\varepsilon, \varepsilon) e^{-\varepsilon \partial_{\varepsilon}}\left(\frac{\varepsilon}{\left(K+\phi_{u}+\varepsilon\right)}\left(\phi_{u}-\delta \phi_{u}\right) c \partial c\right)\right\rangle=-2 \beta+2 \beta-0=0
$$

This is a prescription-independent (and subtraction-independent) result, the reason being that the overall $s$ integrand has, in the limit $\varepsilon \rightarrow 0$, the right convergent behaviour for large $s$ in order to guarantee continuity in $\varepsilon$ also at $\varepsilon=0^{\|}$. We deduce that this is the right way to compute the lump energy, and, as by now should be obvious, the spurious term does not contribute to it.

The term $\Gamma(\varepsilon) \equiv e^{-\varepsilon \partial_{\varepsilon}} \frac{\varepsilon}{K+\phi_{u}+\varepsilon}\left(\phi_{u}-\delta \phi_{u}\right) c \partial c$ in the RHS of (6.20) is clearly similar to the spurious terms considered in connection with the solution to the Einstein EOM in section 3. It violates the principle of continuity and (as a natural consequence) it is ambiguous. However when, in spite of this, it is taken into account in our calculation of the energy outlined in the introduction, it yields a (non-ambiguous) vanishing contribution, as we have just shown, because the integral it is inserted in is convergent (even without the $e^{-\tilde{\eta}_{s}}$ factor). When inserted into non-convergent integrals, in the limit $\varepsilon \rightarrow 0$ it gives rise to an ambiguous term, see (6.13) above. In a well-defined setting, provided by distribution theory, the nature of this term is clear: it is a spurious term and should not be taken into account. In the language of distribution theory, $\phi_{u}^{\varepsilon}$ and $\psi_{u, \varepsilon}$ are not good test states because of their asymptotic behaviors, but their difference is.

\section{Good test string fields}

So far we have seen few example of good test states: one is the state defined implicitly by eq.(6.4), another is in the second line of eq.(6.20) and others, possibly, in the discussion of the CSO. A question one might ask is whether there are enough good test states in the theory. This is connected with the problem of Fock space states. It is customary in SFT to verify a string field's properties by contracting it with Fock space states, the latter being considered a large enough set of states (a completeness). The question of whether $\Gamma(\varepsilon)=e^{-\varepsilon \partial_{\varepsilon}} \frac{\varepsilon}{K+\phi_{u}+\varepsilon}\left(\phi_{u}-\delta \phi_{u}\right) c \partial c$ when contracted with a large enough set of states vanishes must be formulated in the appropriate way. These states cannot be 'naked' Fock space (see some examples of them in [3]) because such states are not good test states. Once again it is worth recalling that if we contract a formally vanishing distribution with a non-test state we can get something nonvanishing. First of all the states we are looking for must be such that the resulting contractions with $\Gamma(\varepsilon)$ be nonsingular (with respect to singularities due to collapsing points). But, especially, they must be characterized by integrable behaviour in the UV and, ignoring the overall $e^{-\varepsilon t}$ factor, in the IR. It is in fact self-evident that all the states with such properties annihilate $\Gamma(\varepsilon)$. The only possibility of getting a nonzero result is linked, as

\footnotetext{
${ }^{\|}$What happens here is that we have the difference of two integrals which are divergent (without the $e^{-\tilde{\eta} s}$ ) but the divergences cancel each other in the limit $\varepsilon \rightarrow 0$.
} 
usual, to correlators characterized by IR linearly divergent integrals (without the exponential $e^{-\varepsilon t}$ ). The question we have to ask is whether there are 'enough' such states. We would like to show in the sequel that they are plentiful.

Consider states created by multiple products of the factor $H\left(\phi_{u}, \varepsilon\right)=\frac{1}{K+\phi_{u}+\varepsilon}\left(\phi_{u}-\delta \phi_{u}\right)$ and contract them with $\Gamma(\varepsilon)$. More precisely, let us define

$$
\Psi_{n}\left(\phi_{u}, \varepsilon\right)=H\left(\phi_{u}, \varepsilon\right)^{n-1} B c \partial c H\left(\phi_{u}, \varepsilon\right) B c \partial c B, \quad n \geq 2
$$

Contracting with $\Gamma(\varepsilon):\left\langle\Psi_{n}\left(\phi_{u}, \varepsilon\right), \Gamma(\varepsilon)\right\rangle$, we obtain a correlator whose IR and UV behaviour (before the $e^{-\varepsilon \partial_{\varepsilon}} \varepsilon$ operator is applied) is not hard to guess. The correlators take the form

$$
\int_{0}^{\infty} d s s^{n} e^{-\tilde{\eta} s} g(s) \int \prod_{i=1}^{n} d x_{i} \varepsilon\left(\left(-\frac{\partial g(s)}{g(s)}\right)^{n+1}+\ldots+\left(-\frac{\partial g(s)}{g(s)}\right)^{n-k+1} G_{s}^{k}+\ldots+G_{s}^{n+1}\right)
$$

where the notation is the same as in section $1.1(s=2 u T)$, but we have tried to make it as compact as possible. The angular variables $x_{i}$ have been dropped in $\mathcal{E}$ and $G_{s}$ (see, for instance, (1.19) where they are explicitly written down). Using the explicit form of $G_{s}(1.20)$, expanding the latter with the binomial formula and integrating over the angular variables, one gets

$$
\int \prod_{i=1}^{n} d x_{i} \varepsilon G_{s}^{k}=\sum_{l=0}^{k} \frac{1}{s^{k-l}} \sum_{n_{1}, \ldots, n_{l}} \frac{P_{l}\left(n_{1}, \ldots, n_{l}\right)}{Q_{l}\left(n_{1}, \ldots, n_{l}\right)} \prod_{i=1}^{l} \frac{1}{p_{i}\left(n_{1}, \ldots, n_{l}\right)+s}
$$

the label $l$ counts the number of cosine factors in each term. Here $n_{i}$ are positive integral labels which come from the discrete summation in $G_{s} ; p_{i}\left(n_{1}, \ldots, n_{l}\right)$ are polynomials linear in $n_{i}$. Next, $P_{l}$ and $Q_{l}$ are polynomials in $n_{i}$ which come from the integration in the angular variables. Every integration in $x_{i}$ increases by 1 the difference in the degree of $Q_{l}$ and $P_{l}$, so that generically $\operatorname{deg} Q_{l}-$ $\operatorname{deg} P_{l}=n$. But in some subcases the integration over angular variables give rise to Kronecker deltas among the indices, which may reduce the degree of $Q_{l}$. So actually the relation valid in all cases is $\operatorname{deg} Q_{l} \geq \operatorname{deg} P_{l}$, but one has to take into account that the number of angular variables to be summed over decreases accordingly.

We are now in the condition to analyze the UV behaviour of (7.2). Let us consider, for instance, the first piece

$$
\sim \int_{0}^{\infty} d s e^{-\tilde{\eta} s} s^{n} g\left(\frac{s}{2}\right)\left(\frac{\partial_{s} g\left(\frac{s}{2}\right)}{g\left(\frac{s}{2}\right)}\right)^{n+1}
$$

Since in the UV $g\left(\frac{s}{2}\right) \approx \frac{1}{\sqrt{s}}$, it is easy to see that the UV behaviour of the overall integrand is $\sim s^{-\frac{3}{2}}$, independently of $n$. As for the other terms, let us consider in the RHS of (7.3) the factor that multiplies $\frac{1}{s^{k-l}}$ (for $l \geq 2$ ). Setting $s=0$, the summation over $n_{1}, \ldots, n_{l-1}$ is always convergent, so that the UV behaviour of each term in the summation is given by the factor $\frac{1}{s^{k-l}}$, with $2 \leq l \leq k$. It follows that the most UV divergent term corresponds to $l=0, \sim \frac{1}{s^{k}}$. Since in (7.2) this is multiplied by

$$
s^{n} g\left(\frac{s}{2}\right)\left(-\frac{\partial g\left(\frac{s}{2}\right)}{g\left(\frac{s}{2}\right)}\right)^{n-k+1}
$$


we see that the UV behaviour of the generic term in (7.2) is at most as singular as $\sim s^{-\frac{3}{2}}$. In conclusion the states $\Psi_{n}$, when contracted with $\Gamma(\varepsilon)$, give rise to the same kind of UV singularity $\sim s^{-\frac{3}{2}}$. Now, for any two such states, say $\Psi_{n}$ and $\Psi_{n^{\prime}}$, we can form a suitable combination such that the UV singularity cancels. In this way we generate infinite many states, say $\Phi_{n}$, which, when contracted with $\Gamma(\varepsilon)$, give rise to UV convergent correlators.

Let us consider next the IR properties $(s \gg 1)$. All the correlators contain the factor $e^{-\tilde{\eta} s}$ which renders them IR convergent, but we have learnt that the crucial IR properties (in the limit $\varepsilon \rightarrow 0$ ) are obtained by ignoring this exponential factor. So, in analyzing the IR properties we will ignore this factor. The first term (7.4) is very strongly convergent in the IR, because $\partial_{s} g\left(\frac{s}{2}\right) \approx \frac{1}{s^{2}}$, while $g\left(\frac{s}{2}\right) \rightarrow 1$. For the remaining terms let us consider in the RHS of (7.3) the factor that multiplies $\frac{1}{s^{k-l}}$ (for $l \geq 2$ ). To estimate the IR behaviour it is very important to know the degree difference between the polynomials $Q_{l}$ and $P_{l}$. Above we said that this difference is always nonnegative. In principle it could vanish, but from the example with $n=2$, see [2], we know that there are cancellations and that in fact the difference in degree is at least 2. If this is so in general, we can conclude that the IR behaviour of the summation in the RHS of (7.3) with fixed $l$ is $\sim \frac{1}{s^{l}}$. However, in order to prove such cancellations, one would have to do detailed calculations, which we wish to avoid here. So we will take the pessimistic point of view and assume that, at least for some of the terms, $\operatorname{deg} Q_{l}=\operatorname{deg} P_{l}$ (in which case there remains only one angular integration). In this case the IR behaviour of the corresponding term cannot decrease faster than $\sim \frac{1}{s^{l-1}}$. This has to be multiplied by $\sim \frac{1}{s^{k-l}}$ and by the IR behaviour of (7.5). This means that the least convergent term with fixed $k$ in(7.3) behaves as $\sim \frac{1}{s^{n-k+1}}$. Since $k \leq n+1$, we see that in the worst hypothesis in the integral (7.2) there can be linearly divergent terms, before the $e^{-\varepsilon \partial_{\varepsilon}} \varepsilon$ operator is applied. If this is so the UV converging $\Phi_{n}$ states are not good test states. However we can repeat for the IR singularities what we have done for the UV ones. Taking suitable differences of the $\Phi_{n}$ 's (this requires a two steps process, first for the linear and then for the logarithmic IR singularities**), we can create an infinite set of states, $\Omega_{n}$, which, when contracted with $\Gamma(\varepsilon)$, yield, before the application of $e^{-\varepsilon \partial_{\varepsilon}} \varepsilon$, a finite result. Upon applying $e^{-\varepsilon \partial_{\varepsilon}} \varepsilon$ they of course vanish. These are therefore good (and nontrivial) test states and, on applying $e^{-\varepsilon \partial_{\varepsilon}} \varepsilon$, they give 0 , i.e. such $\Omega_{n}$ annihilate $\Gamma(\varepsilon)$.

We remark that in eq.(7.1) the presence of $\varepsilon$ in $H\left(\phi_{u}, \varepsilon\right)$ is not essential, because in estimating the IR behaviour we have not counted the $e^{-\tilde{\eta} s}$ factor. Using $\frac{1}{K+\phi_{u}}$ everywhere instead of $\frac{1}{K+\phi_{u}+\varepsilon}$, would lead to the same results. This means that contracting the $\Omega_{n}$ states among themselves (keeping the same ghost factor) leads to finite correlators with or without $\varepsilon$. This, together with the property of annihilating $\Gamma(\varepsilon)$, is a distinctive feature of good test states.

The $\Omega_{n}\left(\phi_{u}, 0\right)$ are however only a first set of good test states. One can envisage a manifold of other such states. Let us briefly describe them, without going into too many details. For instance, let us start again from (7.1) and replace the first $H\left(\phi_{u}, 0\right)$ factor with $\frac{1}{K+\phi_{u}+\varepsilon} u X^{2 k}$ (the term $\delta \phi$ can be dropped). In this way we obtain a new state depending on a new integral label $k$. However replacing $X^{2}$ with $X^{2 k}$ is a too rough operation which renders the calculations unwieldy, because it breaks the covariance with respect to the rescaling $z \rightarrow \frac{z}{t}$. It is rather easy to remedy by studying

\footnotetext{
** In the, so far not met, case where a $\log s$ asymptotic contribution appears in the integrand one would need a three step subtraction process.
} 
the conformal transformation of $X^{2 k}$. The following corrected replacements will do:

$$
\begin{aligned}
u X^{2} & \rightarrow u\left(X^{2}+2(\log u+\gamma)\right)=\phi_{u} \equiv \phi_{u}^{(1)} \\
u X^{4} & \rightarrow u\left(X^{4}+12(\log u+\gamma) X^{2}+12(\log u+\gamma)^{2}\right) \equiv \phi_{u}^{(2)} \\
& \cdots \\
u X^{2 k} & \rightarrow u\left(\sum_{i=0}^{k} \frac{(2 k) !}{(2 k-2 i) ! i !}(\log u+\gamma)^{i} X^{2 k-2 i}\right) \equiv \phi_{u}^{(k)}
\end{aligned}
$$

The role of the additional pieces on the RHS is to allow us to reconstruct the derivatives of $g(s)$ in computing the correlators, as was done in [1].

Now let us denote by $\Psi_{n}^{(k)}$ the $n$-th state (7.1) where $\phi_{u}-\delta \phi_{u}$ in the first $H\left(\phi_{u}, 0\right)$ factor is replaced by $\phi_{u}^{(k)}$. Contracting it with $\Gamma(\varepsilon)$ it is not hard to see that the term (7.4) will be replaced by

$$
\sim \int_{0}^{\infty} d s e^{-\tilde{\eta} s} s^{n} g\left(\frac{s}{2}\right)\left(\frac{\partial_{s} g\left(\frac{s}{2}\right)}{g\left(\frac{s}{2}\right)}\right)^{n+k}
$$

with analogous generalizations for the other terms. It is evident from (7.7) that the UV behaviour becomes more singular with respect to (7.4) while the IR one becomes more convergent. This is a general property of all the terms in the correlator. Thus fixing $k$ we will have a definite UV singularity, the same up to a multiplicative factor for all $\Psi_{n}^{(k)}$. Therefore by combining a finite number of them we can eliminate the UV singularity and obtain another infinite set of UV convergent states $\Phi_{n}^{(k)}$ for any $k$. In general they will be IR convergent (IR subtractions may be necessary for $k=2$ ).

It goes without saying that the previous construction can be further generalized by replacing in (7.1) more than one $X^{2}$ factors with higher powers $X^{2 k}$.

Let us end this section by suggesting another set of states that may be used in order to construct good test states with a subtraction procedure as above. Let us consider states containing a certain number of derivatives of $\phi_{u}$

$$
\Psi_{n, k}\left(\phi_{u}, \varepsilon\right)=\frac{1}{\left(K+\phi_{u}+\varepsilon\right)} \frac{1}{(2 u)^{k}} \partial^{k} \phi_{u} \Psi_{n-1}\left(\phi_{u}, \varepsilon\right)
$$

By contracting them with $\Gamma(\varepsilon)$ we obtain correlators that, before applying $e^{-\varepsilon \partial_{\varepsilon}} \varepsilon$, are defined by integrands in which the UV singularities are worse (and depend on $k$ ), while the IR seem to improve by a factor $\sim \frac{1}{s^{k-1}}$ with respect to $\Psi_{n}$. However the derivative $\partial^{k}$, hitting the propagator $G_{s}$, increases the degree of $P_{l}$. The two effects seem eventually to compensate each other, but the exact IR asymptotic behaviour is more difficult to analyze in this case, unlike the previous examples. For this reason we leave these states as a suggestion to be analyzed in the future.

\section{Spurious terms: comments and conclusions}

Let us summarize the results we have found. We think we have abundantly shown in section 6 that the term $\Gamma(\varepsilon) \equiv e^{-\varepsilon \partial_{\varepsilon}} \frac{\varepsilon}{K+\phi_{u}+\varepsilon}\left(\phi_{u}-\delta \phi_{u}\right) c \partial c$ in $(6.4,6.19)$, when inserted in correlators, is either identically vanishing or ambiguous. The first case occurs when it is inserted in a regular correlator, i.e. in a correlator which is convergent even when the factor $e^{-\varepsilon t}$ coming from the Schwinger 
representation of $\frac{1}{K+\phi_{u}+\varepsilon}$ is replaced by 1 , which implies that the resulting integral (with the $e^{-\varepsilon t}$ factor) is continuous at $\varepsilon=0$ ). This shows that our calculation of the energy in $[2,4]$ is not affected by the term $\Gamma(\varepsilon)$, as one might have feared (see section 3). That also means that the Schwinger representation of an inverse is correct, provided it is used in the correct way.

The second case is when the correlator is at least linearly divergent in the IR (meaning that the correlator is divergent when the factor $e^{-\varepsilon t}$ coming from the Schwinger representation of $\frac{1}{K+\phi_{u}+\varepsilon}$ is removed, which implies that the resulting integral (with the $e^{-\varepsilon t}$ factor) is discontinuous at $\varepsilon=0$ ) : the typical situation is represented by eq.(6.13). In this case we need an ad hoc prescription in order to extract a finite value from the integral, finite value which is originated, as we have shown, by multiplying a zero by $\infty$. It is clear that this is not the right way to compute the energy of anything (neither solutions, nor non-solutions)

The formal presence of the term $\Omega_{u}^{\infty}$ in the RHS of (3.8) or of $e^{-\varepsilon \partial_{\varepsilon}} \frac{\varepsilon}{K+\phi_{u}+\varepsilon}$ in the RHS of (6.1) is simply the spy of the fact that we are evaluating the identity (3.2) on a discontinuous correlator. If the correlator's integrand is convergent enough any such addition as $\frac{1}{K+\phi_{u}} \Omega_{u}^{\infty}$ is irrelevant and $\frac{1}{K+\phi_{u}}$ is correctly represented by (3.1). The appearance of $\Omega_{u}^{\infty}$ or $e^{-\varepsilon \partial_{\varepsilon}} \frac{\varepsilon}{K+\phi_{u}+\varepsilon}$ becomes a pathology of the Schwinger representation which may show up if the problem is not formulated in the proper setting. The appropriate setting is that of distribution theory. In this framework the spurious terms are identically vanishing and there are no violations of the equation of motion.

All these conclusions are based on explicit evaluations and are unquestionable. This said, it would be nice to have a general framework for these problems, a formalization of the rules and procedures we have used above that can be applied in general. At the moment, to our best knowledge, the latter does not exist. The analogy with the case illustrated in section 4 has been instrumental in understanding the nature of the lump solution problem; the treatment there was based on the theory of distributions. We do not seem to have an analogous theory in the case of string fields, but no doubt this is the right instrument we need in order to treat the singularity problems inherent in the search for solutions in SFT.

We cannot hope to solve this problem here. But we think we have clarified the issue at least on one example (the relevant example for our present purposes), that is $\Gamma(\varepsilon)$. An ordinary distribution is just a linear continuous functional on a space of test functions. We can heuristically extend this definition to string fields. A string field distribution is a linear functional on the space of test string fields. In the previous section we have introduced a large set of test states. They are well defined and contain as a particular case the good test states mentioned before. When $\Gamma(\varepsilon)$ is evaluated on them it gives 0 . Therefore in distribution theory this expression is identically vanishing. Said otherwise, it is correct to identify $\Gamma(\varepsilon)$ with the zero in distribution theory.

Invoking distribution theory in order to get rid of the spurious terms in the equation of motion (and elsewhere) may seem ad hoc at first sight, but the interpretation in terms of distribution theory provides a consistent regularization we need in order to make sense of ambiguities. As we have pointed out in section 4 , this is a familiar procedure in theoretical physics in order to carefully define various physical solutions. Apart from the example in section 4, brane solutions in supergravity are often characterized by a metric that explodes when we approach the brane location in the transverse direction, as it depends on some negative power of $r, r$ being the transverse distance. However the relevant physical quantities, like the energy density, are finite. There is only one way to give an 
unambiguous meaning to such solutions: it is to interpret them in the framework of distribution theory.

A formalization of the idea of string field distribution (beyond the example of $\Gamma(\varepsilon)$ studied in detail above) is possible, but, as we pointed out above, to our best knowledge the relevant formalism has not been developed so far. Perhaps the right mathematical setting is offered by the vector distribution theory. The theory of vector distributions was developed by Laurent Schwartz, [24]. The basic objects are a topological vector space and the space of test functions. A distributions is a linear continuous map from the latter to the former. More practically we can think of test vector functions as tensor products of ordinary scalar test functions by vectors and a vector distribution as a space dependent vector, while the evaluation on a vector test function is the ordinary scalar product followed by an ordinary integration. In our case the expression $\frac{1}{K+\phi_{u}}$ should be regarded as a vector distribution. It goes without saying that much work has to be done in order to clarify definitions and show applicability of such formalism in the context of SFT.

\section{References}

[1] L. Bonora, C. Maccaferri and D. D. Tolla, Relevant Deformations in Open String Field Theory: a Simple Solution for Lumps, JHEP 1111:107,2011; arXiv:1009.4158 [hep-th].

[2] L. Bonora, S. Giaccari and D. D. Tolla, The energy of the analytic lump solution in SFT, JHEP 08(2011)158. ArXiv:1105.5926 [hep-th]. Erratum: JHEP 04(2012)001

[3] T. Erler and C. Maccaferri, Comments on Lumps from RG flows, arXiv:1105.6057 [hep-th].

[4] L. Bonora, S. Giaccari and D. D. Tolla, Analytic solutions for Dp branes in SFT, JHEP 12(2011)033; arXiv:1106.3914 [hep-th].

[5] E. Witten, Noncommutative Geometry And String Field Theory, Nucl. Phys. B 268 (1986) 253.

[6] A. Sen, “Descent relations among bosonic D-branes," Int. J. Mod. Phys. A 14, 4061 (1999) [arXiv:hep-th/9902105].

[7] A. Sen, "Universality of the tachyon potential," JHEP 9912, 027 (1999) [arXiv:hep-th/9911116].

[8] M. Schnabl, Analytic solution for tachyon condensation in open string field theory, Adv. Theor. Math. Phys. 10 (2006) 433 [arXiv:hep-th/0511286].

[9] Y. Okawa, Comments on Schnabl's analytic solution for tachyon condensation in Witten's open string field theory, JHEP 0604 (2006) 055 [arXiv:hep-th/0603159].

[10] T. Erler and M. Schnabl, A Simple Analytic Solution for Tachyon Condensation, arXiv:0906.0979 [hep-th].

[11] E. Fuchs and M. Kroyter, Analytical Solutions of Open String Field Theory, arXiv:0807.4722 [hep-th].

[12] M. Schnabl, Algebraic solutions in Open String Field Theory - a lightning review, arXiv:1004.4858 [hep-th].

[13] E. Witten, Some computations in background independent off-shell string theory, Phys. Rev. D 47, 3405 (1993) [arXiv:hep-th/9210065].

[14] D. Kutasov, M. Marino and G. W. Moore, Some exact results on tachyon condensation in string field theory, JHEP 0010, 045 (2000) [arXiv:hep-th/0009148]. 
[15] I. Ellwood, Singular gauge transformations in string field theory, JHEP 0905, 037 (2009) [arXiv:0903.0390 [hep-th]].

[16] I. Ellwood and M. Schnabl, Proof of vanishing cohomology at the tachyon vacuum, JHEP 0702 (2007) 096 [arXiv:hep-th/0606142].

[17] L. Rastelli, A. Sen and B. Zwiebach, Star algebra spectroscopy, JHEP 0203, 029 (2002) [arXiv:hep-th/0111281].

[18] H. Hata and T. Kojita, Winding Number in String Field Theory, JHEP 1201, 088 (2012) [arXiv:1111.2389 [hep-th]].

[19] L. Bonora, C. Maccaferri, R. J. Scherer Santos and D. D. Tolla, Ghost story. I. Wedge states in the oscillator formalism, JHEP 0709, 061 (2007) [arXiv:0706.1025 [hep-th]].

[20] L. Bonora, C. Maccaferri, R. J. Scherer Santos and D. D. Tolla, Ghost story. II. The midpoint ghost vertex, JHEP 0911, 075 (2009) [arXiv:0908.0055 [hep-th]].

[21] L. Bonora, C. Maccaferri and D. D. Tolla, Ghost story. III. Back to ghost number zero, JHEP 0911, 086 (2009) [arXiv:0908.0056 [hep-th]].

[22] N. Dunford and J. T. Schwartz, Linear Operators, vol. I,II. John Wiley \& Sons, New York, 1988.

[23] Y. Okawa, Open string states and D-brane tension from vacuum string field theory, JHEP 0207 (2002) 003 [arXiv:hep-th/0204012].

[24] L. Schwartz, Théorie des distributions à valeurs vectorielles.I Ann.Inst.Fourier, 7 (1957) 1-141. Théorie des distributions à valeurs vectorielles.II Ann.Inst.Fourier, 8 (1958) 1-209.

[25] I. M. Guelfand and G. E. Chilov, Les distributions, tome 1. Dunod, Paris 1962. 